\title{
Anderson transition: a novel route to high thermoelectric performance
}

Fabian Garmroudi ( $\nabla$ fabian.garmroudi@tuwien.ac.at )

TU Wien https://orcid.org/0000-0002-0088-1755

\section{Michael Parzer}

Technische Universität Wien

\section{Alexander Riss}

Technische Universität Wien https://orcid.org/0000-0002-9707-8394

\section{Andrei Ruban}

KTH Royal Institute of Technology

\section{Sergii Khmelevskyi}

Technische Universität Wien

\section{Michele Reticcioli}

Universität Wien https://orcid.org/0000-0001-8223-9928

\section{Matthias Knopf}

Technische Universität Wien

\section{Herwig Michor}

Technische Universität Wien https://orcid.org/0000-0003-1642-5946

\section{Andrej Pustogow}

Technische Universität Wien

\section{Takao Mori}

National Institute for Materials Science https://orcid.org/0000-0003-2682-1846

\section{Ernst Bauer}

Technische Universität Wien

\section{Physical Sciences - Article}

Keywords: thermoelectricity, energy efficiency, Anderson transition

Posted Date: September 27th, 2021

DOl: https://doi.org/10.21203/rs.3.rs-929834/v1

License: (c) (1) This work is licensed under a Creative Commons Attribution 4.0 International License. Read Full License 
Version of Record: A version of this preprint was published at Nature Communications on June 23rd, 2022. See the published version at https://doi.org/10.1038/s41467-022-31159-w. 


\title{
Anderson transition: a novel route to high thermoelectric performance
}

\author{
Fabian Garmroudi ${ }^{\mathrm{a}}$, Michael Parzer ${ }^{\mathrm{a}}$, Alexander Riss ${ }^{\mathrm{a}}$, Andrei V. Ruban ${ }^{\mathrm{b}, \mathrm{c}}$, Sergii Khmelevskyi ${ }^{\mathrm{d}}$, Michele \\ Reticcioli $^{\mathrm{e}}$, Matthias Knopf ${ }^{\mathrm{a}}$, Herwig Michor ${ }^{\mathrm{a}}$, Andrej Pustogow ${ }^{\mathrm{a}}$, Takao Mori ${ }^{\mathrm{f}, \mathrm{g}}$, Ernst Bauer ${ }^{\mathrm{a}}$ \\ ${ }^{a}$ Institute of Solid State Physics, Technische Universität Wien, Vienna, Austria \\ ${ }^{b}$ Department of Materials Science and Engineering, KTH Royal Institute of Technology, Stockholm, Sweden \\ ${ }^{c}$ Materials Center Leoben Forschung GmbH, Leoben, Austria \\ ${ }^{d}$ Research Center for the Computational Materials Science and Engineering, Technische Universität Wien, Vienna, Austria \\ ${ }^{e}$ Faculty of Physics, Center for Computational Materials Science, Universität Wien, Vienna, Austria \\ ${ }^{f}$ International Center for Materials Nanoarchitectonics (WPI-MANA), National Institute for Materials Science, Tsukuba, Japan \\ ${ }^{g}$ Graduate School of Pure and Applied Sciences, University of Tsukuba, Tsukuba, Japan
}

\begin{abstract}
Discovered exactly 200 years ago in 1821,1 thermoelectricity is nowadays of global interest as it allows to directly interconvert thermal and electrical energy via the Seebeck/Peltier effect, which could be exploited to enhance energy efficiency ${ }^{2 / 3}$. In their seminal work ${ }^{4}$, Mahan and Sofo mathematically derived the conditions for 'the best thermoelectric' - a delta-distribution-shaped electronic transport function, where charge carriers contribute to transport only in an infinitely narrow energy interval. So far, however, only approximations to this concept were expected to really exist in nature ${ }^{4 / 5}$. Here, we propose as a physical realisation of this scenario the Anderson transition in an impurity band, i.e. the transition from Anderson-localised to extended quantum states ${ }^{6}$. We obtained a significant enhancement and dramatic change of the thermoelectric properties from $p$-type to $n$-type in the stoichiometric Heusler compound $\mathrm{Fe}_{2} \mathrm{VAl}$, which we assign to a narrow region of delocalised electrons in the energy spectrum near the Fermi energy. We achieved this through an innovative approach of driving the Anderson transition via continuous disorder tuning: variable amounts of atomic defects are induced in a controlled fashion by thermal quenching from high temperatures $\left(950-1380^{\circ} \mathrm{C}\right)$. Based on our experimental electronic transport and magnetisation results, supported by Monte-Carlo and density functional theory calculations, we demonstrate a universal enhancement strategy towards colossal thermoelectric performance that is applicable to diverse material classes.
\end{abstract}

Thermoelectric (TE) devices are capable of converting wasted heat into useful electrical energy or act as Peltier coolers. Facing an increasing worldwide demand for efficient energy utilisation, the immense diversity of potential technologocial applications has sparked great interest 213 Still, TE devices are currently restrained in their applicability due to their limited efficiency. The dimensionless figure of merit $Z T=S^{2} \sigma T /\left(\kappa_{\mathrm{e}}+\kappa_{\mathrm{ph}}\right)$, which is closely related to the conversion efficiency, comprises three materialdependent parameters. These are the thermopower $S$, the electrical conductivity $\sigma$ and the thermal conductivity $\kappa$, consisting of a contribution from electrons $\kappa_{\mathrm{e}}$ and phonons $\kappa_{\mathrm{ph}}$, which are the quanta of lattice vibrations. While considerable progress towards achieving high $Z T$ has been achieved so far by reducing $\kappa_{\mathrm{ph}}{ }^{778}$, increasing the electronic part of $Z T$ is a much more formidable, yet necessary task and new exotic concepts for enhancement are required. In 1996, Mahan and Sofo mathematically identified 'the best thermoelectric' as an ideal system, characterised by

${ }^{*}$ Corresponding author an infinitely narrow delta-distribution-shaped transport function $\Sigma(E)^{4}$.

Here, we propose that this seemingly unrealisable mathematical concept becomes actually realised in real materials at the Anderson transition in an impurity band, as predicted theoretically ${ }^{9}$. As sketched in Fig. 1 , such a transition occurs when the number of randomly distributed impurities increases above a critical value $x_{\mathrm{c}}$, known as quantum percolation. Below $x_{\mathrm{c}}$, all impurity states are Anderson-localised due to disorder 10 . A singularity of the transport function occurs at $x_{\mathrm{c}}$ when an infinitesimally small region of states in the density of states (DOS) becomes delocalised. This was explained by Mott in 1967 through the concept of 'mobility edges', which are two critical energies $E_{\mathrm{c}_{1,2}}$ that appear at the centre of an impurity band, separating localised states in the band tails from delocalised, extended states in the centre ${ }^{11}$. Far above $x_{\mathrm{c}}, E_{\mathrm{c}_{1}}$ and $E_{\mathrm{c}_{2}}$ shift towards the band edges, eventually delocalising all impurity states.

Our study is focused on the TE transport properties across the Anderson transition in an impurity band with 


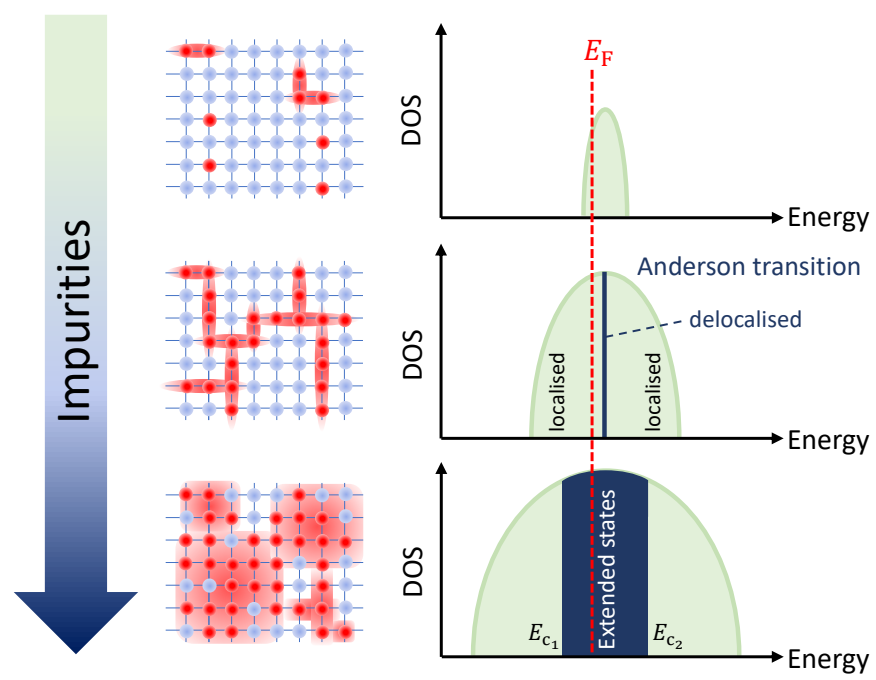

Fig. 1: Schematic of the Anderson transition in an impurity band with increasing impurity concentration. When the number of randomly distributed impurities in a peridocally ordered crystal increases, the impurity electrons remain localised below a threshold value due to Anderson localisation. At the Anderson transition, the critical density of impurities allows for delocalisation of an infinitely narrow energy region of extended states inside the localised impurity states. The delocalised impurity band is marked by two mobility edges $E_{\mathrm{c}_{1,2}}$, which are critical energies that separate the localised from delocalised states. two mobility edges. We experimentally realised such a scenario in undoped, stoichiometric bulk $\mathrm{Fe}_{2} \mathrm{VAl}$ by controlling the degree of lattice disorder via thermal quenching. This Heusler compound recently became an excellent candidate for studying new TE optimisation strategies 12 Our measurements of the electronic transport and magnetisation in this work, supported by Monte-Carlo and density functional theory (DFT) simulations, show clear evidence for a significant enhancement of the TE performance, which we attribute to the Anderson transition. In the following, we describe the structural, electronic and magnetic properties of disorder-tuned $\mathrm{Fe}_{2} \mathrm{VAl}$ as obtained by our experiments and simulations; finally, we show the TE properties of the material at the Anderson transition.

\section{Structural and electronic properties}

Ternary $\mathrm{Fe}_{2} \mathrm{VAl}$ forms a fully ordered $\mathrm{L} 22_{1}$ structure at low temperatures that undergoes two second-order structural phase transitions (see Fig $2 \mathrm{a}$ ) into the partly disordered B2 structure at $T_{\mathrm{B} 2} \approx 1100^{\circ} \mathrm{C}$ and fully disordered A2 structure at $T_{\mathrm{A} 2} \approx 1250^{\circ} \mathrm{C}^{15}$. Our Monte-Carlo simulations based on effective cluster interactions show how the degree of atomic disorder in bulk $\mathrm{Fe}_{2} \mathrm{VAl}$ can be controlled by temperature, finding a remarkable agreement with the experimental $\mathrm{L}_{1}-\mathrm{B} 2$ transition temperature ${ }^{15}$ (the $\mathrm{B} 2-\mathrm{A} 2$ transition temperature was overestimated as dicussed in Methods). This allows us to semiquantitatively assess the concentration of $\mathrm{Fe}, \mathrm{V}$ and $\mathrm{Al}$ atoms on the respective sublattices as depicted in Fig 2b. Note that while the
$\mathrm{V} / \mathrm{Al}$ sublattice is fully disordered in the $\mathrm{B} 2$ phase, there is already a significant site exchange on the Fe sublattice $(5-25 \% \mathrm{Fe}$ antisites). The large amount of antisite defects obtained at high temperatures as a result of the thermal excitations can be partly frozen by ultrafast quenching our samples.

To illustrate the nature of localised $\mathrm{Fe} / \mathrm{V}$ antisite electronic states near the Fermi energy $E_{\mathrm{F}}$, we calculated the spin-polarised DOS by making use of the exact muffin-tin orbital coherent potential approximation method (EMTOCPA). This method allows to calculate the DOS of a singleimpurity embedded in an infinitely large and ordered effective medium, mimicking the electronic and structural properties of an alloy in the dilute limit of antisite concentration $x_{\mathrm{AS}} \rightarrow 0$. Figs 2 , d show the occurence of sharp, hydrogen-like impurity states near $E_{\mathrm{F}}$ for both $\mathrm{Fe}_{\mathrm{V}}$ and $\mathrm{V}_{\mathrm{Fe}}$ defects, as compared to the fully ordered compound. Similar results are obtained for $\mathrm{Fe}_{\mathrm{Al}}$ impurity states (see Extended Data Fig.2). Furthermore, the spin degeneracy
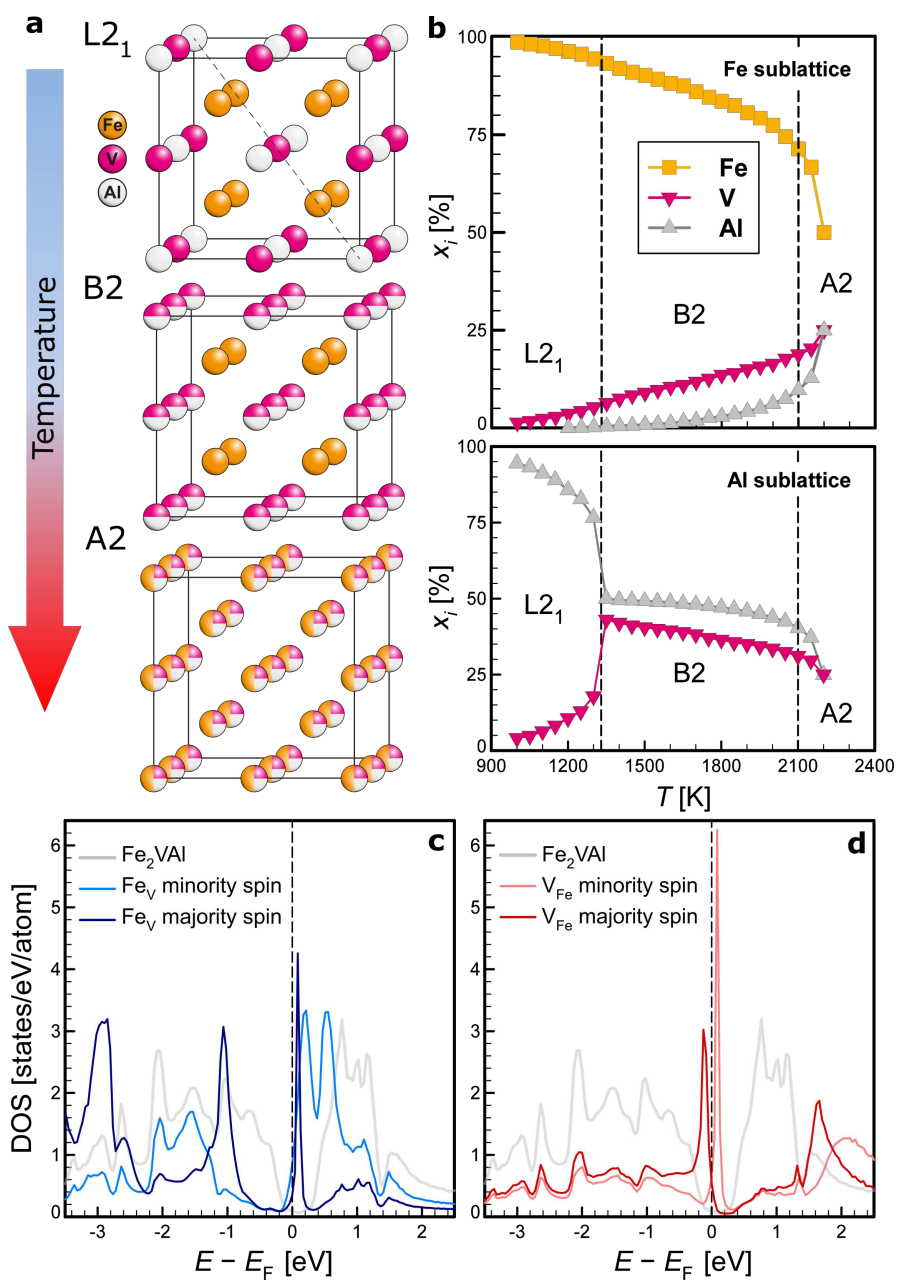

Fig. 2: a, Order-disorder transitions in $\mathrm{Fe}_{2} \mathrm{VAl}$ occuring at high temperatures. b, Monte-Carlo-simulated concentrations of site occupancies in the $\mathrm{L} 2{ }_{1}, \mathrm{~B} 2$ and $\mathrm{A} 2$ high-temperature phases of $\mathrm{Fe}_{2} \mathrm{VAl}$. c,d, Spin-polarised electronic density of states (DOS) for the singleimpurity $\mathrm{Fe}_{\mathrm{V}}$ and $\mathrm{V}_{\mathrm{Fe}}$ antisite defects and pure $\mathrm{Fe}_{2} \mathrm{VAl}$. 
is removed due to the strong correlation of Fe- $d$ electrons, which leads to isolated magnetic impurities in the nonmagnetic, ordered host matrix. With increasing quenching temperature and thus increasing antisite concentration, the randomly distributed isolated defects form a continuum of clusters with different sizes $16[17$, leading to a broadening of the localised electronic states (see Extended Data Fig.3). Eventually, a delocalised impurity band forms, i.e. the Anderson transition, as sketched in Fig 1. However, neither the CPA nor the supercell approach can determine the critical concentration for the Anderson delocalisation transition ${ }^{18}$. To overcome this difficulty, more effortful methods like the typical local density of states calculation ${ }^{18}$ could be used as a means of possibly identifying such transitions in future works.

\section{Magnetic properties}

The formation of magnetic clusters predicted by our simulations shown in the previous section (for details see Methods) can be confirmed by our magnetisation measurements shown in Fig. 3 . Fig 3 a exhibits the field-dependent magnetisation $M$ at $T=4 \mathrm{~K}$ for stoichiometric $\mathrm{Fe}_{2} \mathrm{VAl}$, heat-treated at different conditions. Measurements of the magnetisation have previously shown to be an effective way of probing Anderson-localised states in other semiconductors such as $\mathrm{Si} 19$. The immediate saturation of $M$ at small fields observed in Fig $3 \mathrm{a}$, the absence of hysteresis as well as the strong curvature of isothermal Arrot plots (see Extended Data Fig.7) are strong indications that the magnetic properties are dominated by the localised antisite electrons, in line with our ab initio calculations. In Fig $3 \mathrm{~b}$, we compare the saturation magnetisation $M_{\text {sat }}$ of our samples with their quenching temperature $T_{\text {quench }}$. It can be clearly seen that $M_{\text {sat }}$ consistently increases for higher $T_{\text {quench }}$, corroborating the picture drawn by our ab initio Monte-Carlo calculations. Moreover, both $M_{\text {sat }}$ and the calculated concentration of Fe antisite defects, when rescaled to the experimental transition temperatures, increase in a similar fashion showing an abrupt increase near $T_{\mathrm{A} 2}$. This demonstrates that the rapid quenching method could successfully induce the magnetic antisites in these samples, which is in excellent qualitative agreement with our ab initio calculations.

\section{Thermoelectric properties}

Fig, 4 shows the temperature-dependent electrical resistivity $\rho(T)$ and thermopower $S(T)$ of $\mathrm{Fe}_{2} \mathrm{VAl}$, measured in a wide temperature range from 4 to $800 \mathrm{~K}$ for samples heat-treated and quenched at various temperatures. Above $400-500 \mathrm{~K}$, a semiconductor-like behaviour of the resistivity, $\mathrm{d} \rho / \mathrm{d} T<0$, is found for all samples (see Fig 4 a). This can be attributed to the intrinsic pseudogap of the compound 20121. At lower temperatures, the behaviour modifies from semiconductor-like to metallic, $\mathrm{d} \rho / \mathrm{d} T>0$, demonstrating the Anderson-Mott-type insulator-metal transition
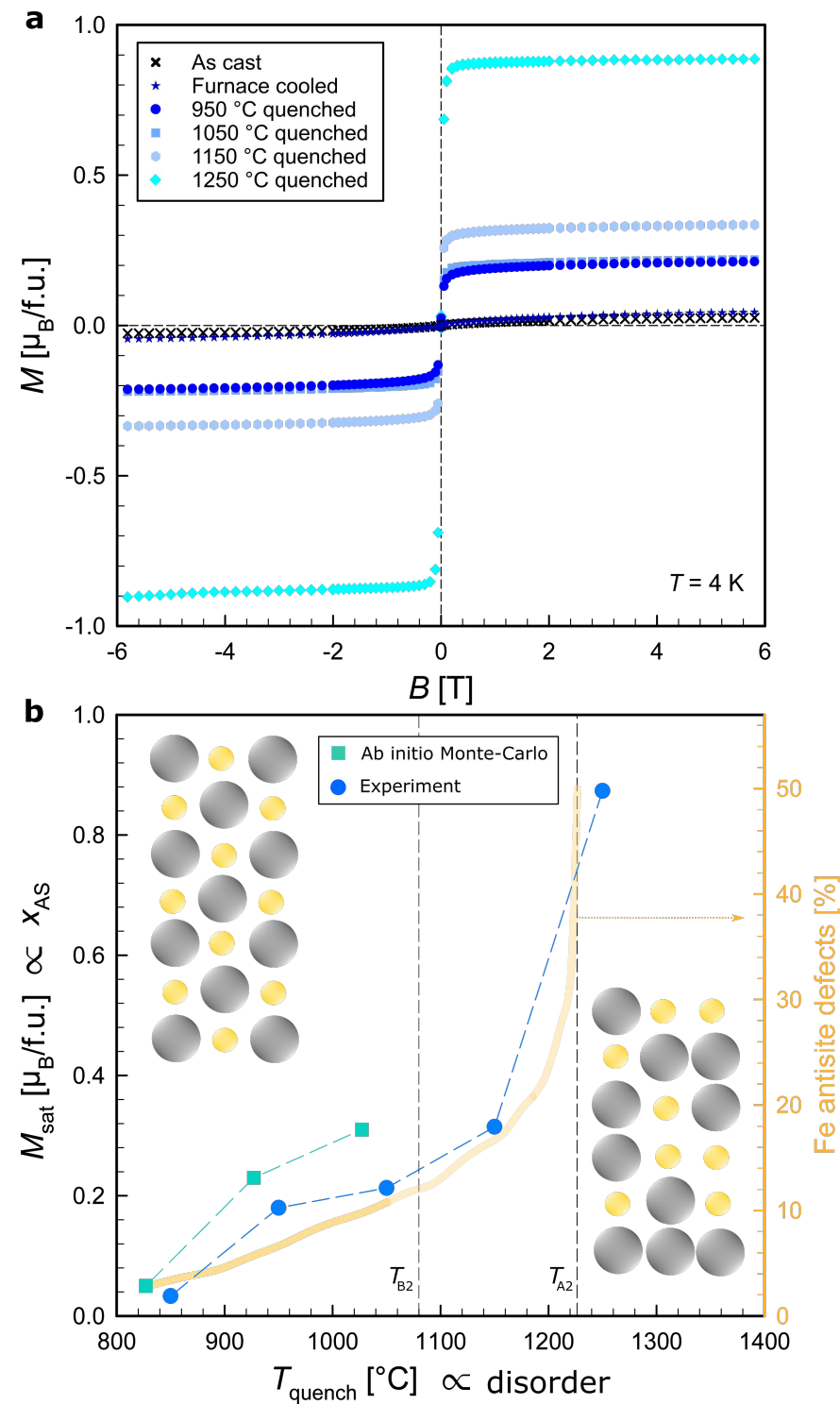

Fig. 3: a, Field-dependent magnetisation of $\mathrm{Fe}_{2} \mathrm{VAl}$ at $T=4 \mathrm{~K}$ for different quenching temperatures. b, Experimental and calculated saturation magnetisation versus quenching temperature. The insets show a sketch of atomic disorder, increasing with $T_{\text {quench }}$. Right scale shows the calculated concentration of Fe antisite defects (yellow curve) from Fig $[1]$, rescaled to the experimental transition temperatures $T_{\mathrm{B} 2}, T_{\mathrm{A} 2} \frac{15}{15}$.

due to the delocalisation of antisite electrons with increas$\operatorname{ing} T_{\text {quench }}$. Indeed, the residual resistivity $\rho_{0}$ decreases by an order of magnitude with increasing $T_{\text {quench }}$, which also manifests itself by a substantial increase of the Hall carrier concentration (see Extended Data Fig.8a), overcompensating the increased number of scattering centres due to disorder. Furthermore, the appearance of metallic transport goes hand in hand with the development of a local maximum in $\rho(T)$ at a temperature $T_{\rho, \max }$, which shifts to higher temperatures as $T_{\text {quench }}$ increases.

In Fig $4 \mathrm{~b}, S(T)$ is shown from 4 to $800 \mathrm{~K}$. As-cast and furnace-cooled samples display positive values of $S(T)$ and a pronounced maximum at $\approx 200 \mathrm{~K}$, consistent with the 

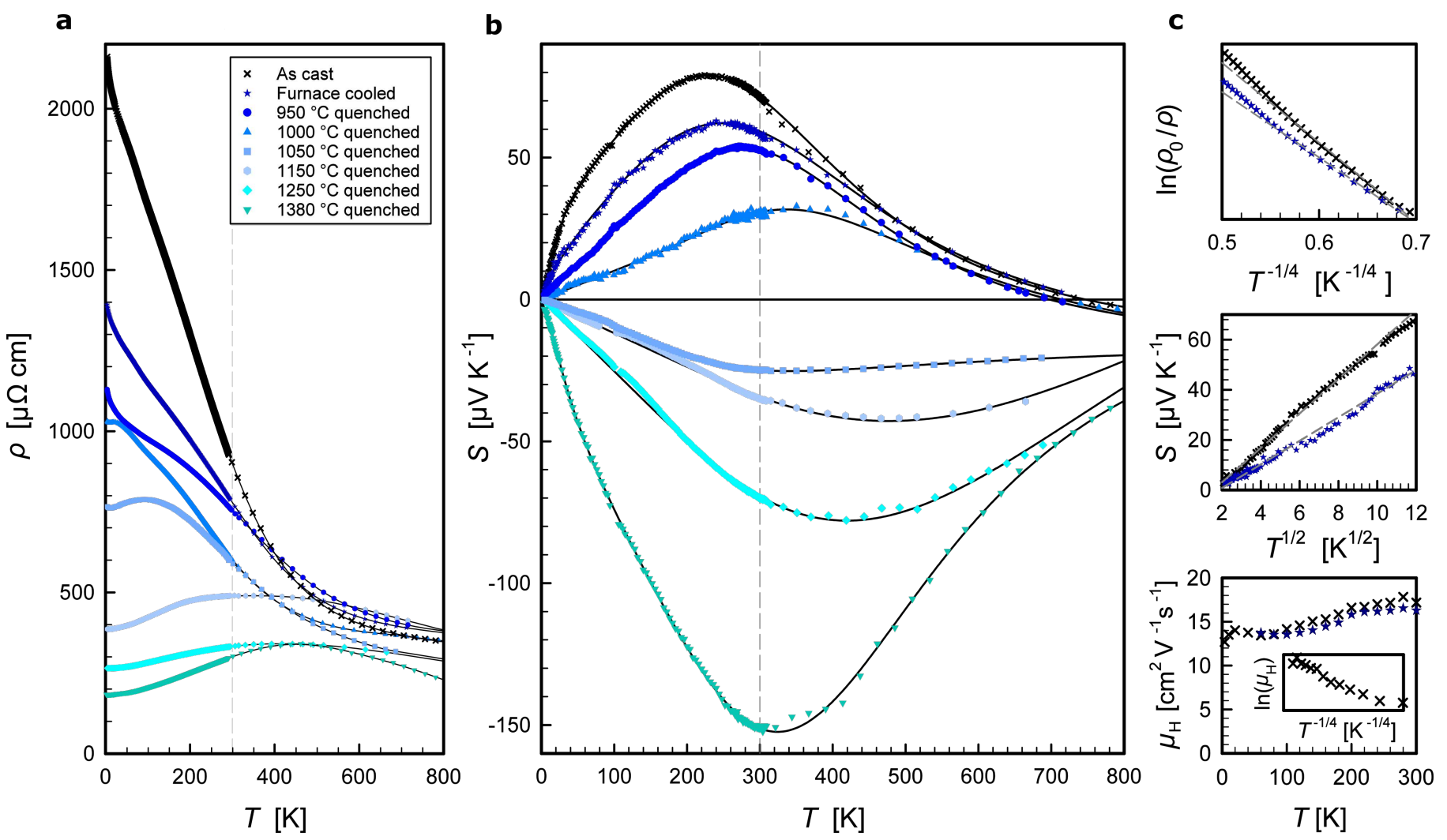

Fig. 4: a, Insulator-metal transition of the temperature-dependent resistivity of Fe 2 VAl with increasing quenching temperature. b, Sign reversal of the temperature-dependent thermopower with increasing quenching temperature. Solid lines are guides to the eye. c, Evidence from different transport measurements for charge localisation and variable-range hopping behaviour of as-cast and furnace-cooled Fe $2 \mathrm{VAl}$ at low temperatures.

narrow pseudogap band structure, where $E_{\mathrm{F}}$ is situated near the valence band edge. As $T_{\text {quench }}$ and the antisite concentration increase, $S(T)$ becomes consistently smaller at all measured temperatures and even exhibits a sign reversal for $T_{\text {quench }}>1000^{\circ} \mathrm{C}$ over the whole temperature range. This implies a substantial negative contribution of the antisite electrons to $S(T)$ in order to account for the dramatic change of the thermopower, from large $p$-type to large $n$-type values.

Further evidence for the presence of Anderson-localised states near $E_{\mathrm{F}}$ is given by a more detailed analysis of the temperature-dependent transport properties. As-cast and furnace-cooled samples, according to the respective magnetic measurements, represent the dilute limit of antisites, where $E_{\mathrm{F}}$ is expected to be situated within the localised states. Indeed, the low-temperature resistivity of these samples (see upper panel Fig,4 can be well described by phonon-assisted variable-range hopping (VRH) conduction 22 :

$$
\rho(T) \propto \exp \left[\left(\frac{T_{0}}{T}\right)^{1 /(d+1)}\right],
$$

which specifies in $3 \mathrm{D}$ to $\rho(T) \propto \exp \left[\left(\frac{T_{0}}{T}\right)^{1 / 4}\right]$, where $T_{0}$ is the characteristic Mott temperature. $T_{0}$ inversely depends on the localisation length $\xi_{\mathrm{L}}$, which diverges at the insulator-metal transition. The fitted values of $T_{0}$ are about $2-4 \mathrm{mK}$, which are at least five orders of magnitude lower than for VRH between localised donor and acceptor states in marginally doped semiconductors ${ }^{2324}$. Again, this low $T_{0}$ corroborates the picture of Anderson-localised states near $E_{\mathrm{F}} 2526$. The temperature-dependent behaviour of the thermopower $S(T) \propto T^{1 / 2}$ at low temperatures (see Fig 4 e centre panel) is also consistent with VRH in $3 \mathrm{D}^{27}$, corroborating the resistivity data. Finally, even the lowtemperature Hall mobility $\mu_{\mathrm{H}}(T)$ shows an unusual, almost constant, slightly increasing temperature dependence (see Fig.4 lower panel), consistent with localisation of charge carriers near $E_{\mathrm{F}} 28$. This picture of Anderson-localised states close to the Fermi level also reconciles many other peculiar properties of this compound, e.g. metallic thermodynamic and photoemission data in spite of semiconductorlike transport properties 20 , negative magnetoresistance ${ }^{20}$, anomalous Hall effect ${ }^{30}$ etc., which have been an ongoing discussion over the past three decades ${ }^{14 \mid 20131}$.

\section{Discussion}

Fig.5 shows in detail the evolution of the electronic transport across the Anderson transition as well as the enormous TE performance that can be attributed to the contribution of the delocalised impurity band. In Fig 5 a we plotted the 

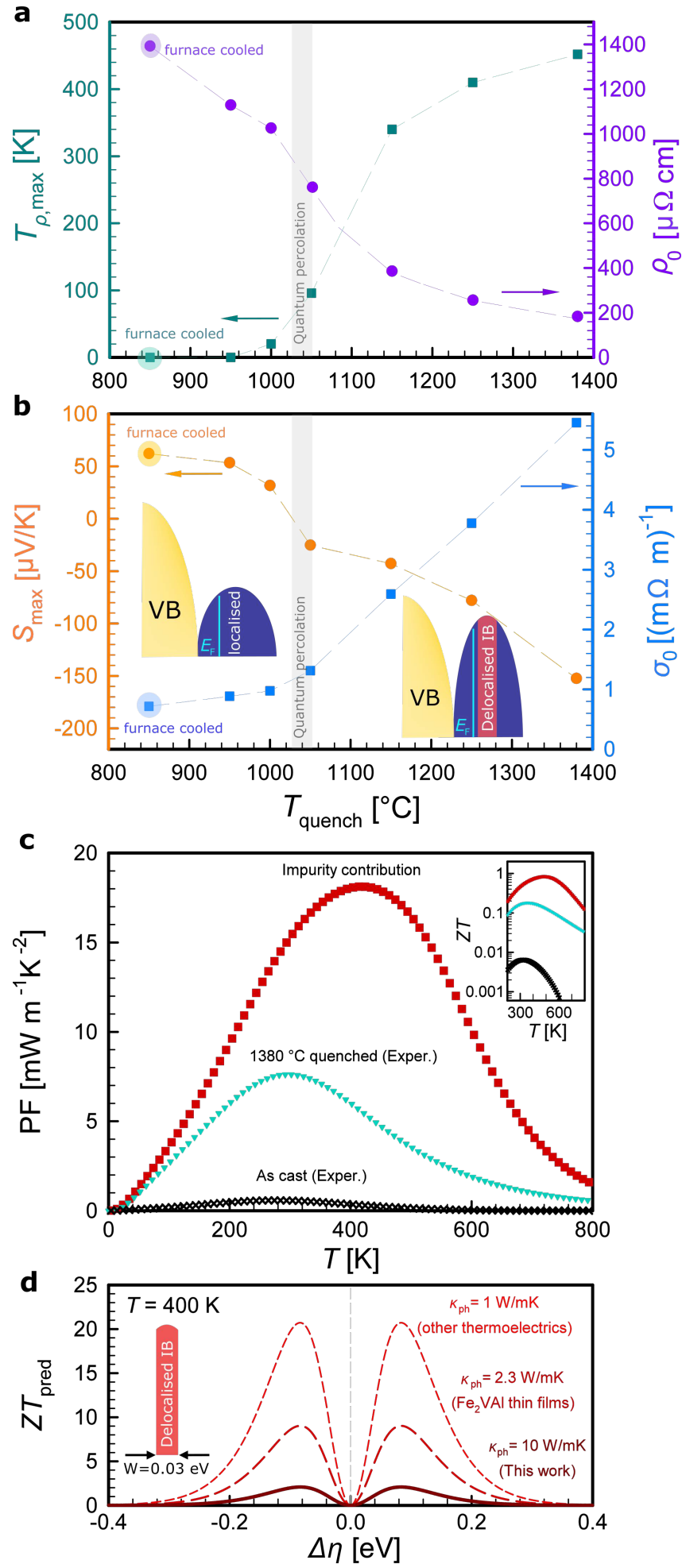

Fig. 5: a, Residual resistivity and temperature of the maximum of the resistivity versus quenching temperature. $\mathbf{b}$, Peak values of the thermopower and residual conductivity versus quenching temperature. c, Power factor of as-cast and $1380^{\circ} \mathrm{C}$-quenched $\mathrm{Fe}_{2} \mathrm{VAl}$ with the contribution of the impurity band (IB). The inset shows the figure of merit $Z T$. d, Predicted $Z T$ versus reduced chemical potential relative to the centre of the impurity band. Model predictions were calculated for different values of the lattice thermal conductivity as explained in Methods. residual resistivity $\rho_{0}$ at $4 \mathrm{~K}$ and $T_{\rho, \max }$ as a function of $T_{\text {quench}}$. With the spontaneous appearance of $T_{\rho, \max }>0$ around $T_{\text {quench }}^{*}=1000-1050{ }^{\circ} \mathrm{C}, \rho_{0}$ simultaneously shows a pronounced kink. In Fig $5 \mathrm{~b}$, we show the peak values of the thermopower $S_{\max }$ as well as the residual conductivity $\sigma_{0}$. Again around $T_{\text {quench }}^{*}, S(T)$ displays a sign reversal and $\sigma_{0}$ deviates from a linear scaling behaviour. These anomalies, together with the monotonous increase of $T_{\rho \text {, max }}$ and decrease of $\rho_{0}$, clearly indicate the continous delocalisation of the impurity band (IB), as sketched in the insets of Fig 5 p.

Fig. 5 shows the power factor $(\mathrm{PF})$ of as-cast and $1380{ }^{\circ} \mathrm{C}$-quenched $\mathrm{Fe}_{2} \mathrm{VAl}$. Usually, the optimisation of thermoelectric materials involves changing the position of the Fermi level, i.e. the total number of electrons, while leaving the electronic structure unchanged, which is called rigid-band doping. Despite being undoped and not yet optimised, the maximum power factor of $1380^{\circ} \mathrm{C}$-quenched $\mathrm{Fe}_{2} \mathrm{VAl}$ is already $7.6 \mathrm{~mW} / \mathrm{mK}^{2}$, which is an enhancement by an order of magnitude compared to the as-cast sample and $40 \%$ higher than the best PF for optimised rigid-band doping in this system 32 . Considering that the total thermopower $S_{\text {tot }}$ and total conductivity $\sigma_{\text {tot }}$ in a material with multiple electronic bands can be written as

$$
\begin{aligned}
S_{\mathrm{tot}} & =\frac{\sum_{i} S_{i} \sigma_{i}}{\sum_{i} \sigma_{i}}, \\
\sigma_{\mathrm{tot}} & =\sum_{i} \sigma_{i},
\end{aligned}
$$

with $S_{i}, \sigma_{i}$ being the respective single-band contributions, we can estimate the contribution of the impurity band which led to the dramatic change in TE transport. Here, the index $(i=\{$ pristine, impurity $\})$ refers to contributions from the pristine band structure and the delocalised impurity band. Bearing in mind that the transport properties of the as-cast sample with purely localised impurity states is mainly dominated by the pristine band structure, we can calculate the additional delocalised impurity contribution to the high-temperature-quenched samples $S_{\mathrm{imp}}, \sigma_{\mathrm{imp}}$ from our measured data by solving the system of Eqs 23. The contribution of the impurity band to the total measured power factor of the $1380^{\circ} \mathrm{C}$-quenched sample is plotted by red squares in Fig 5. An extremely large PF of more than $18 \mathrm{~mW} / \mathrm{mK}^{2}$ at $400 \mathrm{~K}$ is found for a stoichiometric sample not yet optimally doped, exceeding that of the pristine compound by a factor of 30 . It is noteworthy to mention that $\kappa_{\text {ph }}$ was also reduced by a factor of $2-3$ due to the disorder introduced by quenching (see Extended Data Fig.8b). Consequently, this means that the disorder induced by thermal quenching could be a strategy that can enhance all thermoelectric properties at the same time, which is not achievable by conventional doping strategies. We expect that $Z T$ should be further greatly enhanced by optimising the position of $E_{\mathrm{F}}$ and by reducing the background DOS of $\mathrm{Fe}_{2} \mathrm{VAl}$, which can be achieved by appropriate 
co-substitutions with e.g. Si and $\mathrm{Ta}^{30 \mid 32}$.

To make a prediction about potential $Z T$ values in such systems near the Anderson transition, we developed a charge transport model for two mobility edges in an impurity band (see Extended Data Fig.9a). By least-squaresfitting $S(T)$ and $\rho(T)$ of $1380{ }^{\circ} \mathrm{C}$-quenched $\mathrm{Fe}_{2} \mathrm{VAl}$, we found that a narrow impurity band, with a bandwidth of $0.03 \mathrm{eV}$, accounts best for the measured temperature dependencies (see Extended Data Fig.9b). The predictions of the model are shown in Fig $5 \mathrm{~d}$. They reveal that if the chemical potential is placed optimally near the mobility edge, a colossal $Z T>9$ could be achieved if $\kappa_{\mathrm{ph}} \approx 2.3 \mathrm{Wm}^{-1} \mathrm{~K}^{-1}$, currently achievable in $\mathrm{Fe}_{2} \mathrm{VAl}$ by thin film deposition Considering that this strategy might be well applicable to other thermoelectric materials, which generally have even smaller values $\kappa_{\mathrm{ph}} \approx 1 \mathrm{Wm}^{-1} \mathrm{~K}^{-1}$ or lower, huge values of the figure of merit $Z T \gtrsim 20$ are within reach. Such performances in terms of $Z T$ excel those of current state-of-the-art thermoelectrics by an order of magnitude, paving the way for a paradigm shift in energy and cooling applications.

In summary, we theoretically and experimentally demonstrated how the interplay of electronic correlation, Anderson localisation and defect thermodynamics can significantly boost the TE performance in undoped bulk $\mathrm{Fe}_{2} \mathrm{VAl}$ by passing across the Anderson transition, where electrons are delocalised but occupy only a narrow interval in energy space. Indeed, we showed that Mahan and Sofo's 'best thermoelectric' is not just a mathematical construct, but can be implemented in real materials by exploiting charge carriers at the Anderson transition in an impurity band. Moreover, controlling the level of disorder allows us to tune the optimal width of the energy-dependent transport function directly, which is not achievable by other band engineering strategies employed so far. Although disorder and charge localisation have been mostly considered as detrimental for thermoelectricity, our work discloses a novel paradigm to improve thermoelectric materials and devices via impurity conduction, employing temperature-induced disorder as a new tuning and control parameter.

[1] Seebeck, T. J. Ueber den Magnetismus der galvanischen Kette (1822).

[2] Champier, D. Thermoelectric generators: A review of applications. Energy Conversion and Management 140, 167-181 (2017).

[3] Pourkiaei, S. M. et al. Thermoelectric cooler and thermoelectric generator devices: A review of present and potential applications, modeling and materials. Energy 186, 115849 (2019).

[4] Mahan, G. \& Sofo, J. The best thermoelectric. Proceedings of the National Academy of Sciences 93, 7436-7439 (1996).

[5] Heremans, J. P., Wiendlocha, B. \& Chamoire, A. M. Resonant levels in bulk thermoelectric semiconductors. Energy 8 Environmental Science 5, 5510-5530 (2012).

[6] Mott, N. F., Pepper, M., Pollitt, S., Wallis, R. \& Adkins, C. The anderson transition. Proceedings of the Royal Society of London. A. Mathematical and Physical Sciences 345, 169-205 (1975).

[7] Snyder, G. J. \& Toberer, E. S. Complex thermoelectric materials. Materials for Sustainable Energy: A Collection of Peer-Reviewed Research and Review Articles from Nature Publishing Group 101-110 (2011).
[8] Kanatzidis, M. G. Nanostructured thermoelectrics: the new paradigm? Chemistry of Materials 22, 648-659 (2010).

[9] Yamamoto, K., Aharony, A., Entin-Wohlman, O. \& Hatano, N. Thermoelectricity near Anderson localization transitions. Physical Review B 96, 155201 (2017).

[10] Anderson, P. W. Absence of diffusion in certain random lattices. Physical Review 109, 1492 (1958).

[11] Mott, N. Electrons in disordered structures. Advances in Physics 16, 49-144 (1967).

[12] Hinterleitner, B. et al. Thermoelectric performance of a metastable thin-film Heusler alloy. Nature 576, 85-90 (2019).

[13] Tsujii, N., Nishide, A., Hayakawa, J. \& Mori, T. Observation of enhanced thermopower due to spin fluctuation in weak itinerant ferromagnet. Science Advances 5, eaat5935 (2019).

[14] Berche, A., Noutack, M. T., Doublet, M.-L. \& Jund, P. Unexpected band gap increase in the $\mathrm{Fe}_{2} \mathrm{VAl}$ Heusler compound. Materials Today Physics 13, 100203 (2020).

[15] Maier, S. et al. Order-disorder transitions in the $\mathrm{Fe}_{2} \mathrm{VAl}$ Heusler alloy. Acta Materialia 121, 126-136 (2016).

[16] Shklovskii, B. I. \& Efros, A. L. Electronic Properties of Doped Semiconductors, vol. 45 (Springer Science \& Business Media, 2013).

[17] Belitz, D. \& Kirkpatrick, T. The Anderson-Mott transition. Reviews of Modern Physics 66, 261 (1994).

[18] Dobrosavljević, V., Pastor, A. \& Nikolić, B. K. Typical medium theory of Anderson localization: A local order parameter approach to strong-disorder effects. EPL (Europhysics Letters) 62, 76 (2003).

[19] Mori, T., Shimazu, Y. \& Ikehata, S. Magnetic susceptibility of compensated Si:P. Solid State Communications 91, 13-15 (1994).

[20] Nishino, Y. et al. Semiconductorlike behavior of electrical resistivity in Heusler-type $\mathrm{Fe}_{2} \mathrm{VAl}$ compound. Physical Review Letters 79, 1909 (1997).

[21] Okamura, H. et al. Pseudogap formation in the intermetallic compounds $\left(\mathrm{Fe}_{1-x} \mathrm{~V}_{x}\right)_{3} \mathrm{Al}$. Physical Review Letters 84, 3674 (2000).

[22] Mott, N. F. Conduction in non-crystalline materials: III. Localized states in a pseudogap and near extremities of conduction and valence bands. Philosophical Magazine 19, 835-852 (1969).

[23] Prati, E., Hori, M., Guagliardo, F., Ferrari, G. \& Shinada, T. Anderson-Mott transition in arrays of a few dopant atoms in a silicon transistor. Nature nanotechnology 7, 443-447 (2012).

[24] Oliveira, J. F. et al. Pressure-induced Anderson-Mott transition in elemental tellurium. Communications Materials 2, 1-10 (2021).

[25] Sefat, A. S. et al. Anderson-Mott transition induced by hole doping in $\mathrm{Nd}_{1-x} \mathrm{TiO}_{3}$. Physical Review B 74, 104419 (2006).

[26] Ying, T. et al. Anderson localization of electrons in single crystals: $\mathrm{Li}_{x} \mathrm{Fe}_{7} \mathrm{Se}_{8}$. Science Advances 2, e1501283 (2016).

[27] Zvyagin, I. On the theory of hopping transport in disordered semiconductors. Physica Status Solidi (b) 58, 443-449 (1973).

[28] Friedman, L. \& Pollak, M. The Hall effect in the variablerange-hopping regime. Philosophical Magazine B 44, 487-507 (1981).

[29] Vasundhara, M., Srinivas, V. \& Rao, V. Evidence for cluster glass behavior in $\mathrm{Fe}_{2} \mathrm{VAl}$ Heusler alloys. Physical Review B 78, 064401 (2008).

[30] Garmroudi, F. et al. Boosting the thermoelectric performance of $\mathrm{Fe}_{2}$ VAl-type Heusler compounds by band engineering. Physical Review B 103, 085202 (2021).

[31] Weht, R. \& Pickett, W. Excitonic correlations in the intermetallic $\mathrm{Fe}_{2}$ VAl. Physical Review B 58, 6855 (1998).

[32] Kato, H., Kato, M., Nishino, Y., Mizutani, U. \& Asano, S. Effect of silicon substitution on thermoelectric properties of Heusler-type $\mathrm{Fe}_{2} \mathrm{VAl}$ alloy. Nippon Kinzoku Gakkaishi (1952) 65, 652-656 (2001).

[33] Furuta, Y., Kato, K., Miyawaki, T., Asano, H. \& Takeuchi, T. $\mathrm{Fe}_{2}$ VAl-based thermoelectric thin films prepared by a sputtering technique. Journal of Electronic Materials 43, 2157-2164 (2014). 


\section{Supplementary Files}

This is a list of supplementary files associated with this preprint. Click to download.

- MethodsXExtendeddata.pdf 